\title{
Risk of epilepsy in type 1 diabetes mellitus: a population-based cohort study
}

\author{
I-Ching Chou ${ }^{1,2}$ - Chung-Hsing Wang ${ }^{1,3,4}$ - Wei-De Lin ${ }^{5,6}$ • Fuu-Jen Tsai ${ }^{1,3,5,7,8}$. \\ Che-Chen Lin $^{4,9}$ • Chia-Hung Kao ${ }^{10,11}$
}

Received: 9 November 2015 / Accepted: 26 February 2016 /Published online: 31 March 2016

(C) Springer-Verlag Berlin Heidelberg 2016

\begin{abstract}
Aims/hypothesis Type 1 diabetes mellitus is a major public health problem of increasing global concern, with potential neurological complications. A possible association exists between type 1 diabetes and subsequent epilepsy. This study evaluated the relationship between type 1 diabetes and epilepsy in Taiwan. Methods Claims data from the Taiwan National Health Insurance Research Database were used to conduct retrospective cohort analyses. The study cohort contained 2568 patients with type 1 diabetes, each of whom was frequency-matched by sex, urbanisation of residence area and index year with ten patients without type 1 diabetes. Cox proportional hazard regression analysis was conducted to estimate the effects of type 1 diabetes on epilepsy risk.

Results In patients with type 1 diabetes, the risk of developing epilepsy was significantly higher than that in patients without type 1 diabetes $(p<0.0001$ for logrank test). After adjustment for potential confounders, the type 1 diabetes cohort was 2.84 times as likely to develop epilepsy than the control cohort was (HR 2.84 [95\% CI 2.11, 3.83]).
\end{abstract}

Chia-Hung Kao

d10040@mail.cmuh.org.tw

1 Department of Pediatric Neurology, China Medical University Children's Hospital, Taichung, Taiwan

2 Graduate Institute of Integrated Medicine, College of Chinese Medicine, China Medical University, Taichung, Taiwan

3 Department of Pediatric Genetics, China Medical University Children's Hospital, Taichung, Taiwan

4 School of Medicine, China Medical University, Taichung, Taiwan

5 Department of Medical Research, China Medical University Hospital, Taichung, Taiwan
Conclusions/interpretation Patients with type 1 diabetes are at an increased risk of developing epilepsy. Metabolic abnormalities of type 1 diabetes, such as hyperglycaemia and hypoglycaemia, may have a damaging effect on the central nervous system and be associated with significant long-term neurological sequelae. The causative factors between type 1 diabetes and the increased risk of epilepsy require further investigation.

Keywords Epilepsy $\cdot$ National Health Insurance Research Database $\cdot$ Type 1 diabetes

$\begin{array}{ll}\text { Abbreviations } \\ \text { ICD-9-CM } & \text { ICD-9, clinical modification } \\ \text { IRB } & \text { Institutional Review Board } \\ \text { LBW } & \begin{array}{l}\text { Low birthweight } \\ \text { (Taiwan) National Health Insurance } \\ \text { NHI }\end{array} \\ & \begin{array}{l}\text { (programme) } \\ \text { NHIRD }\end{array} \\ & \text { Insurance National Health } \\ \end{array}$

6 School of Post Baccalaureate Chinese Medicine, China Medical University, Taichung, Taiwan

7 School of Chinese Medicine, China Medical University, Taichung, Taiwan

8 Department of Health and Nutrition Biotechnology, Asia University, Taichung, Taiwan

9 Management Office for Health Data, China Medical University Hospital, Taichung, Taiwan

10 Graduate Institute of Clinical Medical Science and School of Medicine, College of Medicine, China Medical University, No. 2, Yuh-Der Road, Taichung 40447, Taiwan

11 Department of Nuclear Medicine and PET Center, China Medical University Hospital, Taichung, Taiwan 


\section{Introduction}

Type 1 diabetes mellitus is one of the most common autoimmune disorders in children, with a $3 \%$ annual increase in the global incidence rate since the 1980s [1-3]. In recent decades, the incidence of type 1 diabetes has increased in children and adolescents, particularly those aged younger than 5 years [4]. Patients with diabetes are at increased risk of severe health problems and mortality $[5,6]$.

Epilepsy is a common serious neurological condition that is characterised by a predisposition to epileptic seizures and that has significant effects on the social and behavioural development of children [7, 8]. The causes of epilepsy can be broadly categorised as structural abnormalities, metabolic disturbances and genetic factors. However, these causes account for only $25-45 \%$ of all cases, and there is no clear underlying cause in the remaining population, thus representing forms of idiopathic epilepsy [9].

Recent studies have found that type 1 diabetes could be a risk factor for the development of epilepsy in children, though the exact underlying mechanisms remain unknown. Metabolic abnormalities of diabetes, such as hyperglycaemia and hypoglycaemia, may have a damaging effect on the central nervous system [10], thereby leading to seizure. Specific autoimmune causes typically associated with autoantibodies have been increasingly identified in a subset of previously idiopathic seizure disorders [11-17].

According to our review of the literature, data on longterm neurological outcomes in children with type 1 diabetes are limited. Few studies have epidemiologically analysed the association between type 1 diabetes and epilepsy. McCorry et al found a significant surplus of type 1 diabetes in an idiopathic generalised epilepsy cohort, with an OR of 4.4 [18]. However, O'Connell et al could not confirm these results; after examining the type of epilepsy and prevalence in children and adolescents with type 1 diabetes, they found no difference compared with those in the general paediatric population [19].

In the current study, we used population-based data from the Taiwan National Health Insurance Research Database (NHIRD) to test the hypothesis that type 1 diabetes increases the risk of epilepsy.

\section{Methods}

Data source This study used the NHIRD to construct the study population. The NHIRD contains all claims data from the Taiwan National Health Insurance (NHI) programme, a compulsory and universal single-payer health insurance programme into which 13 health insurance programmes have been incorporated since 1995. The coverage rate of the NHI reached $99 \%$ of the 23 million residents of Taiwan in 1998.
The NHIRD is renewed annually and includes registries for beneficiaries, disease diagnosis records, medical prescriptions and other medical services. To ensure privacy, the original identification numbers of insurants are replaced with anonymous surrogate identification codes. This study was deemed to fulfil the conditions for exemption of review by the Institutional Review Board (IRB) of China Medical University (CMUH104-REC2-115). The IRB also waived the consent requirement.

In the NHIRD, all disease diagnosis records are listed according to the ICD-9, clinical modification (ICD-9-CM; www. icd9data.com/2007/Volume1). The history of type 1 diabetes was collected from the catastrophic illness registry. The epilepsy diagnosis and comorbidity history of each study patient was collected from inpatient and outpatient files.

Study population To investigate the association between the risk of epilepsy and type 1 diabetes, we conducted a retrospective population-based cohort study. The type 1 diabetes cohort comprised patients aged $\leq 18$ years who were diagnosed between 1 January 2000 and 31 December 2009 as having newonset type 1 diabetes according to the ADA diagnostic criteria (ICD-9-CM 250.01, 250.11, 250.13, 250.21, 250.23, 250.31, $250.33,250.41,250.43,250.51,250.53,250.61,250.63$, $250.71,250.73,250.81,250.83,250.91$ and 250.93; Table 1) $[20,21]$. The index date for the type 1 diabetes patients was the initial date of type 1 diabetes diagnosis. The controls were patients without type 1 diabetes diagnosis in the NHIRD and were frequency-matched with the type 1 diabetes patients by sex and urbanisation at a 10:1 ratio. For their index dates, the comparison patients were randomly assigned a month and date with the same index year as that of the matched cases. The major outcome of the study was epilepsy occurrence (ICD-9-CM 345). Follow-up was terminated when the patient withdrew from the NHI programme, when epilepsy occurred, or on 31 December 2011.

Comorbidities considered confounders were prior epilepsy, head injury (ICD-9-CM 850-854 and 959.01), intellectual disabilities (ICD-9-CM 317-319) and low birthweight (LBW, ICD-9-CM 765). Urbanisation was classified into four levels. Level 1 indicated the highest urbanisation level, whereas level 4+ represented the lowest level. Urbanisation level was determined according to indices including population density (people $/ \mathrm{km}^{2}$ ) and the population ratios of different educational levels, elderly people and agricultural workers, as well as the number of physicians per 100000 people [22]. Urbanisation was recategorised as urban (urbanisation levels 1 and 2) and rural (urbanisation levels 3 and 4+).

We also considered hypoglycaemia status. A patient was deemed to have hypoglycaemia if an in-hospital diagnosis (ICD-9-CM 251.0-251.2 and 775.6) occurred during follow-up. 
Table 1 ICD-9-CM codes and descriptions used for selection of type 1 diabetes cases

\begin{tabular}{ll}
\hline ICD-9-CM & Description \\
\hline 250.01 & Type 1 diabetes mellitus without mention of complication \\
$250.11,250.13$ & Type 1 diabetes with ketoacidosis \\
$250.21,250.23$ & Type 1 diabetes with hyperosmolarity \\
$250.31,250.33$ & Type 1 diabetes with other coma \\
$250.41,250.43$ & Type 1 diabetes with renal manifestations \\
$250.51,250.53$ & Type 1 diabetes with ophthalmic manifestations \\
$250.61,250.63$ & Type 1 diabetes with neurological manifestations \\
$250.71,250.73$ & Type 1 diabetes with peripheral circulatory disorders \\
$250.81,250.83$ & Type 1 diabetes with other specified manifestations \\
$250.91,250.93$ & Type 1 diabetes with unspecified complication \\
\hline
\end{tabular}

Statistical analysis To present the relationship between the type 1 diabetes cohort and comparison cohort, age was presented as mean (SD), and sex, urbanisation and comorbidities were assigned a number and percentage. A $t$ test and $\chi^{2}$ test was used to determine the differences for continuous and categorical variables, respectively. The incidence density of epilepsy for the study population was calculated according to the total number of epilepsy events divided by the sum of follow-up years (per 10000 person-years). The cumulative incidence curves for the two study cohorts were measured using the Kaplan-Meier method. To estimate the risk of epilepsy in patients with type 1 diabetes, we used single-variable and multivariable Cox proportional hazard models to measure the HRs and 95\% CIs. We also used stratified analysis to determine the epilepsy risk in patients with type 1 diabetes according to different levels of demographic factors and comorbidities.

Data management and statistical analysis were performed using SAS 9.4 software (SAS Institute, Cary, NC, USA). The plots of cumulative incidence curves were drawn using $\mathrm{R}$ software (R Foundation for Statistical Computing, Vienna, Austria). The significance level was set at $p<0.05$ for twosided testing.

\section{Results}

We collected 2,568 patients with type 1 diabetes and 25,680 comparison patients (Table 2). Both cohorts were $46.5 \%$ male, and nearly $60 \%$ of the study patients lived in highly urbanised areas (urbanisation levels 1 and 2). The mean age of the patients with type 1 diabetes was 10.4 years, and the mean age of the comparison cohort was 0.7 years older than that of the type 1 diabetes cohort. The proportions of intellectual disabilities and prior epilepsy in the type 1 diabetes cohort were significantly greater than those in the comparison cohort $(p<0.05)$, whereas the proportions of head injury and LBW were not.

The incidences of epilepsy were 10.4 and 33.7 per 10,000 person-years for the comparison and type 1 diabetes patients, respectively (Table 3 ). The cumulative incidence of epilepsy after a 12-year follow-up measured using the Kaplan-Meier method was $2.2 \%$ greater in the type 1 diabetes cohort than in the comparison cohort (logrank test, $p<0.0001$; Fig. 1). After adjustment for age, sex, urbanisation level, prior epilepsy, intellectual disabilities, LBW and head injury, the risk of epilepsy in the type 1 diabetes patients was 2.84 -fold that in those without type 1 diabetes (HR 2.84 [95\% CI 2.11, 3.83]). The main effects of age, sex and urbanisation were not statistically significant for the risk of epilepsy. Patients with prior epilepsy had a dramatically increased risk of epilepsy (HR 53.7[95\% CI 40.4, 71.5]). Intellectual disability was also significantly

Table 2 Baseline demographics and comorbidities of comparison and type 1 diabetes cohorts

\begin{tabular}{llll}
\hline Variable & $\begin{array}{l}\text { Comparison } \\
\text { cohort } \\
N=25,680\end{array}$ & $\begin{array}{l}\text { T1DM cohort } \\
N=2,568\end{array}$ & $p$ value $^{\mathrm{a}}$ \\
& $11.1 \pm 4.3$ & $10.4 \pm 4.4$ & $<0.0001$ \\
\hline Age, years & $3,563 \pm 13.9$ & $508 \pm 19.8$ & \\
$\quad<6$ & $10,354 \pm 40.3$ & $990 \pm 38.6$ & \\
$6-11$ & $11,763 \pm 45.8$ & $1,070 \pm 41.7$ & \\
$\geq 12$ & & & $>0.99$ \\
Sex & $13,740(53.5)$ & $1,374(53.5)$ & \\
$\quad$ Female & $11,940(46.5)$ & $1,194(46.5)$ & \\
$\quad$ Male & & & $>0.99$ \\
Urbanisation & $7,370(28.7)$ & $737(28.7)$ & \\
1 & $8,030(31.3)$ & $803(31.3)$ & \\
2 & $4,550(17.7)$ & $455(17.7)$ & \\
3 & $5,730(22.3)$ & $573(22.3)$ & \\
$\quad 4+$ & & & \\
Comorbidity & & $41(1.6)$ & 0.0248 \\
$\quad$ Prior epilepsy & $283(1.1)$ & $84(3.3)$ & 0.4834 \\
Head injury & $776(3.0)$ & $33(1.3)$ & 0.0006 \\
Intellectual disability & $175(0.7)$ & $23(0.9)$ & 0.7283 \\
LBW & $248(1.0)$ & & \\
\hline
\end{tabular}

Data are means \pm SD or $n(\%)$

${ }^{\text {a }} p$ values were calculated using a $t$ test

T1DM, type 1 diabetes 
Table 3 Incidence of epilepsy and HRs for study cohort, determined using multivariate Cox proportional hazards regression analysis

\begin{tabular}{|c|c|c|c|c|c|}
\hline Variable & Event & PYs & Rate & $\operatorname{HR}(95 \% \mathrm{CI})$ & Adjusted $\mathrm{HR}^{\mathrm{a}}(95 \% \mathrm{CI})$ \\
\hline \multicolumn{6}{|l|}{ T1DM } \\
\hline No & 181 & 17,4229 & 10.4 & Ref. & Ref. \\
\hline Yes & 59 & 17,522 & 33.7 & $3.26(2.43,4.37)$ & $2.84(2.11,3.83)$ \\
\hline \multicolumn{6}{|l|}{ Age group } \\
\hline$<6$ & 55 & 36,162 & 15.2 & Ref. & Ref. \\
\hline $6-11$ & 85 & 75,222 & 11.3 & $0.66(0.47,0.93)$ & $0.60(0.42,0.84)$ \\
\hline$\geq 12$ & 100 & 80,367 & 12.4 & $0.71(0.51,0.99)$ & $0.67(0.48,0.95)$ \\
\hline \multicolumn{6}{|l|}{ Sex } \\
\hline Female & 114 & 102,389 & 11.1 & Ref. & Ref. \\
\hline Male & 126 & 89,362 & 14.1 & $1.27(0.98,1.63)$ & $1.12(0.87,1.45)$ \\
\hline \multicolumn{6}{|l|}{ Urbanisation } \\
\hline 1 & 76 & 54,675 & 13.9 & Ref. & Ref. \\
\hline 2 & 76 & 59,962 & 12.7 & $0.91(0.67,1.26)$ & $0.83(0.61,1.15)$ \\
\hline 3 & 38 & 33,991 & 11.2 & $0.81(0.55,1.19)$ & $0.82(0.55,1.21)$ \\
\hline $4+$ & 50 & 43,122 & 11.6 & $0.84(0.59,1.20)$ & $0.80(0.56,1.14)$ \\
\hline \multicolumn{6}{|l|}{ Prior epilepsy } \\
\hline No & 152 & 190,302 & 7.99 & Ref. & Ref. \\
\hline Yes & 88 & 1,450 & 607 & $68.1(52.3,88.7)$ & $53.7(40.4,71.5)$ \\
\hline \multicolumn{6}{|l|}{ Head injury } \\
\hline No & 220 & 186,485 & 11.8 & Ref. & Ref. \\
\hline Yes & 20 & 5,266 & 38.0 & $3.08(1.95,4.88)$ & $1.91(1.20,3.05)$ \\
\hline \multicolumn{6}{|c|}{ Intellectual disability } \\
\hline No & 215 & 190,646 & 11.3 & Ref. & Ref. \\
\hline Yes & 25 & 1,106 & 226 & $18.4(12.2,27.9)$ & $3.17(2.01,5.01)$ \\
\hline \multicolumn{6}{|l|}{ LBW } \\
\hline No & 233 & 190,187 & 12.3 & Ref. & Ref. \\
\hline Yes & 7 & 1,564 & 44.8 & $3.42(1.61,7.26)$ & $1.88(0.87,4.05)$ \\
\hline
\end{tabular}

${ }^{a}$ Model adjusted for age, sex, urbanisation, prior epilepsy, intellectual disability, LBW and head injury PYs, person-years; rate, incidence rate per 10,000 person-years; T1DM, type 1 diabetes associated with an increased risk of epilepsy. Table 4 provides the data of the study population without a history of epilepsy before the index date; the results reveal that the risk of

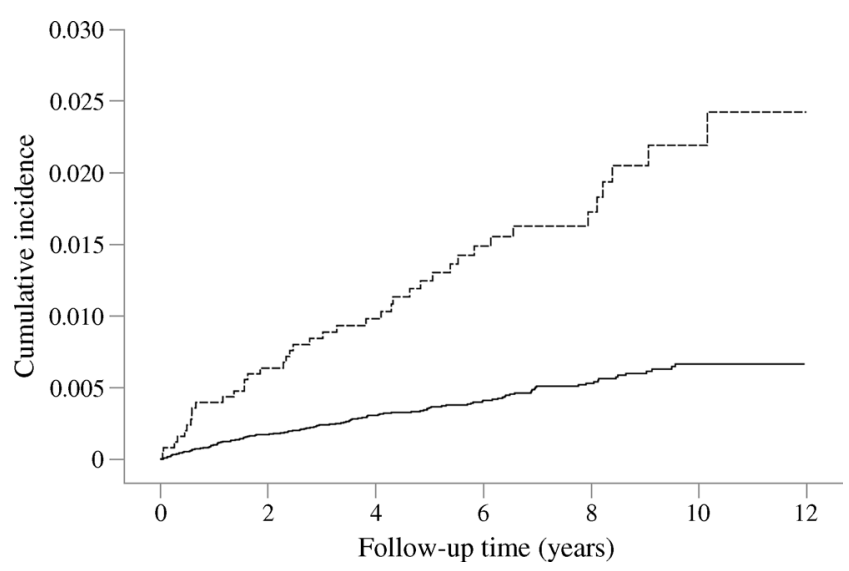

Fig. 1 Epilepsy incidence curves for patients with type 1 diabetes (dashed line) and the comparison cohort (solid line). The difference between the curves was significant (logrank test, $p<0.0001$ ) epilepsy in patients with type 1 diabetes was nearly 3.36 -fold $(95 \%$ CI $2.35,4.81)$ that in the comparison cohort.

Table 5 shows the epilepsy risk in the patients with type 1 diabetes stratified by demographic factors and comorbidities. We observed that the incidence of epilepsy was greater in the type 1 diabetes cohort than in the comparison cohort according to comorbidity status and demographic factors. Because of the small study population with comorbidities, the epilepsy risk was no different between the patients with and without type 1 diabetes regarding head injury, prior epilepsy, intellectual disability or LBW. The patients with type 1 diabetes exhibited a significantly increased risk of epilepsy compared with the comparison patients according to age $<6$ years (HR $6.06[95 \%$ CI 3.41, 10.8]), age 6-11 years (HR 1.86[95\% CI $1.06,3.24]$ ), age $\geq 12$ years (HR $2.47[95 \%$ CI $1.52,4.03]$ ), being male (HR2.54[95\% CI 1.66, 3.89]) or female (HR 3.58 [95\% CI 2.33, 5.50]) and living in urban (HR $2.47[95 \%$ CI $1.68,3.62]$ ) or rural areas (HR 3.61[95\% CI 2.24, 5.82]). 
Table 4 Incidence of epilepsy and multivariate Cox proportional hazards regression analysis measured hazard ratio for study cohort both without epilepsy history before index date.

\begin{tabular}{rlllll}
\hline Variable & Event & PYs & Rate & HR $(95 \% \mathrm{CI})$ & $\mathrm{aHR}^{\mathrm{a}}(95 \% \mathrm{CI})$ \\
\hline T1DM & & & & & \\
No & 116 & 171,514 & 6.76 & Ref. & Ref. \\
Yes & 41 & 17,334 & 23.65 & $3.51(2.46,5.01)$ & $3.36(2.35,4.81)$ \\
\hline
\end{tabular}

${ }^{a}$ Model adjusted for age, sex, urbanisation, intellectual disability, low birthweight and head injury

aHR, adjusted HR; PYs, person-years; rate, incidence rate per 10,000 person-years

Table 6 shows the risk of epilepsy in type 1 diabetes patients with and without hypoglycaemia. Relative to the comparison cohort, the HRs of epilepsy were 2.67 (95\% CI 1.97, $3.62)$ and 16.5 (95\% CI 5.19, 52.3) for the type 1 diabetes patients without and with hypoglycaemia, respectively. The results also revealed that the epilepsy risk increased with type 1 diabetes severity ( $p$ for trend $<0.0001$ ).

\section{Discussion}

Critical questions remain regarding the outcomes of type 1 diabetes, including neuropsychological function. In this study, we found that patients with type 1 diabetes are at an increased risk of developing epilepsy (HR2.84[95\%, CI2.11, 3.83]).
This result is consistent with those of previous studies in that epilepsy or seizures are observed in many autoimmune or inflammatory disorders and are linked to the primary disease or secondary to pro-inflammatory processes [11-17]. Moreover, we determined that the proportion of intellectual disabilities in the type 1 diabetes cohort was significantly greater than that in the comparison cohort $(p=0.0006)$. Furthermore, children with an intellectual disability exhibited a significantly increased risk of epilepsy.

The mechanisms of the association between type 1 diabetes and epilepsy remain unclear. Researchers have proposed several hypotheses on the possible pathophysiology of their comorbidity, including immune abnormalities, brain lesions, genetic factors and metabolic abnormalities [23]. In particular, both hyperglycaemia and hypoglycaemia commonly occur in elderly people with diabetes, and can alter the balance between the inhibition and excitation of neuronal networks and cause focal motor seizures [24-26]. In addition, we found that younger age was associated with an increased risk of developing epilepsy. Previous studies have suggested severe hypoglycaemia, young age and early onset as critical risk factors for electroencephalographic abnormalities [10]. Serial electroencephalographic recordings made in 70 children with diabetes revealed that those who had electroencephalographic abnormalities were younger and had an earlier onset of diabetes. In addition, 21 of the 34 with electroencephalographic abnormalities (62\%) had experienced a severe hypoglycaemic attack; however, abnormalities were found in only 13 of the 43
Table 5 Demographic factors and estimated HRs of epilepsy risk in type 1 diabetes and comparison groups, stratified by comorbidities

\begin{tabular}{|c|c|c|c|c|c|c|c|}
\hline \multirow[b]{2}{*}{ Comorbidity } & \multicolumn{3}{|c|}{ Comparison group } & \multicolumn{3}{|c|}{ T1DM group } & \multirow{2}{*}{$\begin{array}{l}\text { Adjusted } \mathrm{HR}^{\mathrm{a}} \\
(95 \% \mathrm{CI})\end{array}$} \\
\hline & Event & PYs & Rate & Event & PYs & Rate & \\
\hline \multicolumn{8}{|l|}{ Age group } \\
\hline$<6$ & 35 & 32,500 & 10.8 & 20 & 3,662 & 54.6 & $6.06(3.41,10.8)$ \\
\hline $6-11$ & 69 & 68,623 & 10.1 & 16 & 6,599 & 24.3 & $1.86(1.06,3.24)$ \\
\hline$\geq 12$ & 77 & 73,106 & 10.5 & 23 & 7,261 & 31.7 & $2.47(1.52,4.03)$ \\
\hline \multicolumn{8}{|l|}{ Sex } \\
\hline Female & 85 & 93,068 & 9.13 & 29 & 9,321 & 31.1 & $3.58(2.33,5.50)$ \\
\hline Male & 96 & 81,161 & 11.8 & 30 & 8,201 & 36.6 & $2.54(1.66,3.89)$ \\
\hline \multicolumn{8}{|l|}{ Urbanisation } \\
\hline Urban & 117 & 104,145 & 11.2 & 35 & 10,493 & 33.4 & $2.47(1.68,3.62)$ \\
\hline Rural & 64 & 70,084 & 9.13 & 24 & 7,030 & 34.1 & $3.61(2.24,5.82)$ \\
\hline \multicolumn{8}{|l|}{ Prior epilepsy } \\
\hline No & 109 & 172,945 & 6.30 & 43 & 17,357 & 24.8 & $3.80(2.67,5.42)$ \\
\hline Yes & 72 & 1,284 & 561 & 16 & 165 & 967 & $1.66(0.96,2.87)$ \\
\hline \multicolumn{8}{|l|}{ Head injury } \\
\hline No & 164 & 169,434 & 9.68 & 56 & 17,051 & 32.8 & $2.97(2.19,4.04)$ \\
\hline Yes & 17 & 4,795 & 35.5 & 3 & 472 & 63.6 & $1.54(0.42,5.67)$ \\
\hline \multicolumn{8}{|c|}{ Intellectual disability } \\
\hline No & 159 & 173,337 & 9.17 & 56 & 17,309 & 32.4 & $3.32(2.45,4.51)$ \\
\hline Yes & 22 & 892 & 247 & 3 & 214 & 140 & $0.72(0.20,2.62)$ \\
\hline \multicolumn{8}{|l|}{ LBW } \\
\hline No & 176 & 172,808 & 10.2 & 57 & 17,379 & 32.8 & $2.70(2.00,3.65)$ \\
\hline Yes & 5 & 1,421 & 35.2 & 2 & 143 & 140 & - \\
\hline
\end{tabular}

${ }^{a}$ Model adjusted for age, sex, urbanisation, prior epilepsy, intellectual disability, LBW and head injury

PYs, person-years; rate, incidence rate per 10,000 person-years; T1DM, type 1 diabetes 
Table 6 Incidence of epilepsy and adjusted HRs of type 1 diabetes patients with and without hypoglycaemia, determined using multivariate Cox proportional hazards regression analysis

\begin{tabular}{lllll}
\hline Variable & Event & PYs & Rate & Adjusted HR ${ }^{\mathrm{a}}(95 \% \mathrm{CI})$ \\
\hline $\begin{array}{l}\text { Comparison cohort } \\
\text { T1DM cohort }\end{array}$ & 181 & 174,229 & 10.4 & ref \\
$\quad$ Without hypoglycaemia & 56 & 17,274 & 32.4 & $2.67(1.97,3.62)$ \\
$\quad$ With hypoglycaemia & 3 & 248 & 121 & $16.5(5.19,52.3)$ \\
$\quad p$ for trend & & & & $<0.0001$ \\
\hline
\end{tabular}

${ }^{\text {a }}$ Model adjusted for age, sex, urbanisation, prior epilepsy, intellectual disability, LBW and head injury PYs, person-years; rate, incidence rate per 10,000 person-years; T1DM, type 1 diabetes children with diabetes who did not have severe hypoglycaemia $(30 \%)$. All the children with diabetes and hypoglycaemic convulsions had permanent electroencephalographic abnormalities [10].

Another hypothesis explaining seizures in patients with type 1 diabetes might be related to specific autoantibodies against neural components. Specific autoimmune causes, typically associated with autoantibodies, have been increasingly identified in a subset of previously idiopathic seizure disorders [11-17]. Epileptologists are becoming increasingly interested in autoimmune processes and in the search for pathogenic antibodies. Recently, Ong et al conducted a population-level study to examine the relationship between epilepsy and 12 common autoimmune diseases: type 1 diabetes, psoriasis, rheumatoid arthritis, Graves' disease, Hashimoto's thyroiditis, Crohn's disease, ulcerative colitis, systemic lupus erythematosus, antiphospholipid syndrome, Sjögren syndrome, myasthenia gravis and coeliac disease. The risk of epilepsy was consistently observed across all 12 autoimmune diseases and exhibited an increased OR of 3.9 in children ( $<18$ years) with type 1 diabetes [27]. The researchers concluded that epilepsy and autoimmune diseases frequently co-occur, and that the potential role of autoimmunity must be accounted for when epilepsy is considered. In particular, GAD antibodies have been associated with type 1 diabetes and a wide variety of neurological conditions, including epilepsy [28-30]. GAD antibodies have been detected in $80 \%$ of patients with a new diagnosis of type 1 diabetes, in approximately $80 \%$ of patients with stiff person syndrome and in some patients with epilepsy [29].

In this study, we found that the proportion of intellectual disabilities was higher in the type 1 diabetes cohort than in the general population. These results were consistent with those of previous studies, in that children with type 1 diabetes were found to be at an increased risk of cognitive and behavioural difficulties [31-33]. Moreover, an uncontrolled pilot study observed an association between improved glycaemic control after continual subcutaneous insulin infusion commencement and improvements in mood, behaviour and performance in complex cognitive tasks [34]. In addition, focal neurological deficits associated with hypoglycaemia have been observed in children with diabetes. One report presented a series of 19 episodes in seven children with diabetes [35], and another study reported focal motor seizures, followed by ipsilateral hemiparesis associated with hypoglycaemia [36]. Although autoimmunity and neuroinflammation are likely to play a role in a subset of epilepsy patients with type 1 diabetes, seizures may also be a result of cerebrovascular complications that are commonly associated with many types of brain damage. Thus, the risk of seizures in epilepsy patients may be independent of immunological causes.

Type 1 diabetes care in Taiwan follows several recognised guidelines for the diagnosis, control and management of diabetes, covering various components of diabetes care, often with an emphasis on glucose-lowering therapies [37]. However, the current study was subject to some limitations that must be mentioned. First, the NHIRD provides no detailed patient information such as on lifestyle habits, BMI, physical activity, socioeconomic status and family history. All of these were possible confounders in this study. Second, the evidence derived from a cohort study is generally of a lower methodological quality than that from randomised trials, because a cohort study design is subject to many biases related to adjustment for confounders. Despite our meticulous study design with adequate control for confounders, a key limitation was that bias could remain because of possible unmeasured or unknown confounders. Third, the NHI claims registries primarily serve the purpose of administrative billing and do not undergo verification for scientific purposes. We were unable to contact the patients directly to obtain more information on their use of drugs or subtype of epilepsy, because of the anonymised identification numbers. However, the data that we obtained on the diagnoses of type 1 diabetes and epilepsy were highly reliable.

In conclusion, our results show that patients with type 1 diabetes exhibit a higher incidence of epilepsy. The pathogenetic mechanisms of neurological diseases remain unknown but may be associated with significant long-term neurological sequelae. The current results could provide evidence to facilitate the prognosis of children with type 1 diabetes. The causative factors between type 1 diabetes and the increased risk of epilepsy require further investigation. 
Funding This study was supported in part by the study project (DMR105-032) in our hospital; Ministry of Science and Technology, R. O. C. (104-2314-B-039-044-MY2); Taiwan Ministry of Health and Welfare Clinical Trial and Research Center of Excellence (MOHW105-TDU-B212-133019); China Medical University Hospital, Academia Sinica Taiwan Biobank, Stroke Biosignature Project (BM10501010037); NRPB Stroke Clinical Trial Consortium (MOST 104-2325-B-039-005); Tseng-Lien Lin Foundation, Taichung, Taiwan; Taiwan Brain Disease Foundation, Taipei, Taiwan; Katsuzo and Kiyo Aoshima Memorial Funds, Japan; and China Medical University (CMU) under the Aim for the Top University Plan of the Ministry of Education, Taiwan. The funders had no role in the study design, data collection and analysis, decision to publish, or preparation of the manuscript. No additional external funding was received for this study.

Duality of interest The authors declare that there is no duality of interest associated with this manuscript.

Author contributions All authors have contributed substantially to, and are in agreement with the content of, the manuscript. I-CC and C$\mathrm{HK}$ are responsible for the conception and design of the study. C-HK provided the study materials. All authors were involved in the collection and/or assembly of data, data analysis and interpretation, and preparation of the manuscript preparation. All authors approved the final version of the manuscript. The guarantor of the paper, taking responsibility for the integrity of the work as a whole, from inception to published article, is C-HK.

\section{References}

1. Knip M, Siljander H (2008) Autoimmune mechanisms in type 1 diabetes. Autoimmun Rev 7:550-557

2. Rawshani A, Landin-Olsson M, Svensson AM et al (2014) The incidence of diabetes among 0-34 year olds in Sweden: new data and better methods. Diabetologia 57:1375-1381

3. Patterson C, Guariguata L, Dahlquist G, Soltész G, Ogle G, Silink M (2014) Diabetes in the young - a global view and worldwide estimates of numbers of children with type 1 diabetes. Diabetes Res Clin Pract 103:161-175

4. Atkinson MA, Eisenbarth GS, Michels AW (2014) Type 1 diabetes. Lancet 383:69-82

5. Kollipara S (2006) Comorbidities associated with type 1 diabetes. School Nurse News 23:42-45

6. Zhao Y, Ye W, Boye KS, Holcombe JH, Swindle R (2009) Healthcare charges and utilization associated with diabetic neuropathy: impact of Type 1 diabetes and presence of other diabetesrelated complications and comorbidities. Diabet Med 26:61-69

7. Moshè SL, Perucca E, Ryvlin P, Tomson T (2015) Epilepsy: new advances. Lancet 7:884-898

8. Fisher RS, Acevedo C, Arzimanoglou A et al (2014) ILAE official report: a practical clinical definition of epilepsy. Epilepsia 55:475482

9. Cowan LD (2002) The epidemiology of the epilepsies in children. Ment Retard Dev Disabil Res Rev 8:171-181

10. Soltész G, Acsádi G (1989) Association between diabetes, severe hypo-glycaemia, and electroencephalographic abnormalities. Arch Dis Child 64:992-996

11. Bergey GK (2012) Autoantibodies in the patient with drugresistant epilepsy: are we missing a treatable etiology? Arch Neurol 69:565-566
12. Palace J, Lang B (2000) Epilepsy: an autoimmune disease? J Neurol Neurosurg Psychiatry 69:711-714

13. McKnight K, Jiang Y, Hart $Y$ et al (2005) Serum antibodies in epilepsy and seizure-associated disorders. Neurology 65:1730 1736

14. Barajas RF, Collins DE, Cha S, Geschwind MD (2010) Adult-onset drug-refractory seizure disorder associated with anti-voltage-gated potassium-channel antibody. Epilepsia 51:473-477

15. Liimatainen S, Peltola M, Sabater L et al (2010) Clinical significance of glutamic acid decarboxylase antibodies in patients with epilepsy. Epilepsia 51:760-767

16. Lin JJ, Lin KL, Hsia SH, Wang HS, Chou IJ, Lin YT (2012) CHEESE Study Group. Antiglutamic acid decarboxylase antibodies in children with encephalitis and status epilepticus. Pediatr Neurol 47:252-258

17. Dalmau J, Gleichman AJ, Hughes EG et al (2008) Anti-NMDAreceptor encephalitis: case series and analysis of the effects of antibodies. Lancet Neurol 7:1091-1098

18. McCorry D, Nicolson A, Smith D, Marson A, Feltbower RG, Chadwick DW (2006) An association between type 1 diabetes and idiopathic generalized epilepsy. Ann Neurol 59:204-206

19. O'Connell MA, Harvey AS, Mackay MT, Cameron FJ (2008) Does epilepsy occur more frequently in children with Type 1 diabetes? J Paediatr Child Health 44:586-589

20. Silverstein J, Klingensmith G, Copeland K et al (2005) Care of children and adolescents with type 1 diabetes: a statement of the American Diabetes Association. Diabetes Care 28:186

21. American Diabetes Association (2011) Standards of medical care in diabetes. Diabetes Care 34(Suppl 1):S11

22. Liu CY, Hung YT, Chuang YL et al (2006) Incorporating development stratification of Taiwan townships into sampling design of large scale health interview survey. J Health Manag 14:1-22

23. Yun C, Xuefeng W (2013) Association between seizures and diabetes mellitus: a comprehensive review of literature. Curr Diabetes Rev 9:350-354

24. Maheandiran M, Mylvaganam S, Wu C et al (2013) Severe hypoglycemia in a juvenile diabetic rat model: presence and severity of seizures are associated with mortality. PLoS One 8:e83168

25. Schwechter EM, Velísková J, Velísek L (2003) Correlation between extracellular glucose and seizure susceptibility in adult rats. Ann Neurol 53:91-101

26. Kirchner A, Velísková J, Velísek L (2006) Differential effects of low glucose concentrations on seizures and epileptiform activity in vivo and in vitro. Eur J Neurosci 23:1512-1522

27. Ong MS, Kohane IS, Cai T, Gorman MP, Mandl KD (2014) Population-level evidence for an autoimmune etiology of epilepsy. JAMA Neurol 71:569-574

28. Striano P, Errichiello L, Striano S (2011) Autoantibodies to glutamic acid decarboxylase in patients with epilepsy: what is their clinical relevance? Epilepsy Behav 20:145

29. Saiz A, Blanco Y, Sabater L et al (2008) Spectrum of neurological syndromes associated with glutamic acid decarboxylase antibodies: diagnostic clues for this association. Brain 131:2553-2563

30. Olson JA, Olson DM, Sandborg C, Alexander S, Buckingham B (2002) Type 1 diabetes mellitus and epilepsy partialis continua in a 6-year-old boy with elevated anti-GAD65 antibodies. Pediatrics 109, E50

31. Brands AM, Biessels GJ, de Haan EH, Kappelle LJ, Kessels RP (2005) The effects of type 1 diabetes on cognitive performance: a meta-analysis. Diabetes Care 28:726-735

32. Desrocher M, Rovet J (2004) Neurocognitive correlates of type 1 diabetes mellitus in childhood. Child Neuropsychol 10:36-52

33. Northam EA, Rankins D, Cameron FJ (2006) Therapy insight: the impact of type 1 diabetes on brain development and function. Nat Clin Pract Neurol 2:78-86 
34. Knight S, Northam E, Donath S et al (2009) Improvements in cognition, mood and behaviour following commencement of continuous subcutaneous insulin infusion therapy in children with type 1 diabetes mellitus: a pilot study. Diabetologia 52:193-198

35. Wayne EA, Dean HJ, Booth F, Tenenbein M (1990) Focal neurological deficits associated with hypoglycemia in children with diabetes. J Pediatr 117:575-577
36. Lahat E, Barr J, Bistritzer T (1995) Focal epileptic episodes associated with hypoglycemia in children with diabetes. Clin Neurol Neurosurg 97:314-316

37. Home P, Haddad J, Latif ZA et al (2013) Comparison of national/ regional diabetes guidelines for the management of blood glucose control in non-Western Countries. Diabetes Ther 4:91-102 